\title{
Remarks on some recent results about polynomials with restricted zeros
}

\begin{abstract}
We point out certain flaws in two papers published in Ann. Univ. Mariae Curie-Skłodowska Sect. A, one in 2009 and the other in 2011. We discuss in detail the validity of the results in the two papers in question.
\end{abstract}

1. Introduction. The following result was proved by Govil [3].

Theorem A. Let $P(z)$ be a polynomial of degree $n$ having all its zeros in the disk $|z| \leq k$ for some $k \geq 1$. Then

$$
\max _{|z|=1}\left|P^{\prime}(z)\right| \geq \frac{n}{1+k^{n}} \max _{|z|=1}|P(z)| .
$$

The result is best possible and equality holds for $P(z)=z^{n}+k^{n}$.

The next result is also due to Govil [4, p. 184, Theorem D].

Theorem B. Let $P(z)=\sum_{k=0}^{n} a_{k} z^{k}$ be a polynomial of degree $n$ having all its zeros on $|z|=k$ for some $k \leq 1$. Then

$$
\max _{|z|=1}\left|p^{\prime}(z)\right| \leq \frac{n}{k^{n}+k^{n-1}} \max _{|z|=1}|p(z)| .
$$

In [2] the authors state and I quote: "In this paper, we consider a class of polynomials $P(z)=c_{0}+\sum_{\nu=\mu}^{n} c_{\nu} z^{\nu}, 1 \leq \mu \leq n$ and generalize as well as

2010 Mathematics Subject Classification. 30A10, 30C10, 30C15.

Key words and phrases. Polynomials, extremal properties, Bernstein's inequality. 
improve upon Theorem A and also generalize Theorem B by proving the following results". They state their "so-called generalizations" of Theorem A, etc. as follows.

Theorem 1. If $P(z)=c_{0}+\sum_{\nu=\mu}^{n} c_{\nu} z^{\nu}, 1 \leq \mu<n$ is a polynomial of degree $n$, having all its zeros in the disk $|z| \leq k, k \geq 1$, then

$$
\max _{|z|=1}\left|P^{\prime}(z)\right| \geq \frac{n}{1+k^{n-\mu+1}} \max _{|z|=1}|P(z)| .
$$

The result is best possible and equality holds for

$$
P(z)=\left(z^{n-\mu+1}+k^{n-\mu+1}\right)^{\frac{n}{n-\mu+1}} .
$$

Theorem 2. If $P(z)=c_{n} z^{n}+\sum_{\nu=\mu}^{n} c_{n-\nu} z^{n-\nu}, 1 \leq \mu<n$ is a polynomial of degree $n$, having all its zeros on $|z|=k, k \leq 1$, then

$$
\begin{aligned}
& \max _{|z|=1}\left|P^{\prime}(z)\right| \\
& \quad \leq \frac{n}{k^{n-\mu+1}}\left(\frac{n\left|c_{n}\right| k^{2 \mu}+\mu\left|c_{n-\mu}\right| k^{\mu-1}}{\mu\left|c_{n-\mu}\right|\left(1+k^{\mu-1}\right)+n\left|c_{n}\right| k^{\mu-1}\left(1+k^{\mu+1}\right)}\right) \max _{|z|=1}|P(z)| .
\end{aligned}
$$

Theorem 3. If $P(z)=c_{0}+\sum_{\nu=\mu}^{n} c_{\nu} z^{\nu}, 1 \leq \mu<n$ is a polynomial of degree $n$, having all its zeros in the disk $|z| \leq k, k \geq 1$, then

$$
\max _{|z|=1}\left|P^{\prime}(z)\right| \geq \frac{n}{1+k^{n-\mu+1}}\left\{\max _{|z|=1}|P(z)|+\min _{|z|=k}|P(z)|\right\} .
$$

The result is best possible and equality holds for

$$
P(z)=\left(z^{n-\mu+1}+k^{n-\mu+1}\right)^{\frac{n}{n-\mu+1}} .
$$

2. Some comments on Theorems 1, 2 and 3. Unfortunately, Theorems 1 and 3 are false. As regards Theorem 2, its proof is based on a lemma that is erroneous.

To see that Theorem 1 is false, let us consider the example $P(z):=z^{n}+k^{n}$. This is a polynomial which does have the form $c_{0}+\sum_{\nu=\mu}^{n} c_{\nu} z^{\nu}, 1 \leq \mu<n$ with

$$
c_{0}=k^{n}, c_{\nu}=0 \text { for } \nu=\mu, \ldots, n-1 \text {, and } c_{n}=1 \text {, }
$$

where $\mu$ can be taken to be any integer in $\{1,2, \ldots, n-1\}$. Besides, it has all its zeros on $|z|=k$. Clearly, $\max _{|z|=1}|P(z)|=1+k^{n}$ and $\max _{|z|=1}\left|P^{\prime}(z)\right|=$ $n$. Thus, if (3) was true, then we would have

$$
n \geq \frac{n}{1+k^{n-\mu+1}}\left(1+k^{n}\right)
$$

for any $\mu \in\{1, \ldots, n-1\}$, which amounts to saying that $k^{n-\mu+1} \geq k^{n}$ for any $\mu \in\{1, \ldots, n-1\}$. For $k>1$, this is obviously false except when $\mu=1$. Even if $k^{n-\mu+1}=k^{n}$ when $\mu=1$ or $k=1$, it is of no significance since when $\mu=1$ or $k=1$, the so-called Theorem 1 says nothing more than what Theorem A does. 
In the face of this counter-example, the authors of [2] might claim that in $z^{n}+k^{n}$, which is our counter-example, the coefficients $c_{1}, \ldots, c_{n-1}$ are all zero whereas in Theorem $1, c_{\mu}$ is supposed to be different from 0 . So, we shall give a counter-example in which $c_{\mu} \neq 0$.

Take any $a>1$ and consider the polynomial $P(z):=z^{n}+\delta z^{\mu}+a^{n}$, where $\delta$ is supposed to be positive and small. Since the zeros of $P$ are continuous functions [5, p. 9] of $\delta$ and those of $z^{n}+a^{n}$ all lie on $|z|=a$ the polynomial $P$ has all its zeros in $|z| \leq k$, where $|k-a| \rightarrow 0$ as $\delta \rightarrow 0$. Now, note that

$$
\max _{|z|=1}|P(z)|=1+\delta+a^{n} \text { and } \max _{|z|=1}\left|P^{\prime}(z)\right|=n+\delta \mu .
$$

Then, according to Theorem 1, we would have

$$
\left(\mu-\frac{n}{1+k^{n-\mu+1}}\right) \delta \geq\left(\frac{1+a^{n}}{1+k^{n-\mu+1}}-1\right) n=\left(\frac{a^{n}-k^{n-\mu+1}}{1+k^{n-\mu+1}}\right) n .
$$

As $\delta \rightarrow 0$,

$$
\left(\frac{a^{n}-k^{n-\mu+1}}{1+k^{n-\mu+1}}\right) n \rightarrow\left(\frac{a^{n}-a^{n-\mu+1}}{1+a^{n-\mu+1}}\right) n,
$$

which is strictly positive if $1<\mu<n-1$. Hence, for any such $\mu$, there exists a positive number $\delta_{0}$ such that

$$
\left(\frac{a^{n}-k^{n-\mu+1}}{1+k^{n-\mu+1}}\right) n>\frac{1}{2}\left(\frac{a^{n}-a^{n-\mu+1}}{1+a^{n-\mu+1}}\right) n \text { for } 0<\delta<\delta_{0} .
$$

Now, from (5) it follows that

$$
\left(\mu-\frac{n}{1+k^{n-\mu+1}}\right) \delta>\frac{1}{2}\left(\frac{a^{n}-a^{n-\mu+1}}{1+a^{n-\mu+1}}\right) n
$$

for $0<\delta<\delta_{0}$. This cannot be true since the expression on the lefthand side of the inequality tends to 0 as $\delta \rightarrow 0$ whereas the expression on the right-hand side is a positive constant. The second sentence in the statement of Theorem 1 is: "The result is best possible and equality holds for $P(z)=\left(z^{n-\mu+1}+k^{n-\mu+1}\right)^{\frac{n}{n-\mu+1}}$ ". This statement implicitly presumes that $\left(z^{n-\mu+1}+k^{n-\mu+1}\right)^{n /(n-\mu+1)}$ is a polynomial. However, for $\left(z^{n-\mu+1}+\right.$ $\left.k^{n-\mu+1}\right)^{n /(n-\mu+1)}$ to be a polynomial, $n$ must be divisible by $n-\mu+1$. Surprisingly, the authors do not seem to realize this. This remark also applies to the second sentence in the statement of Theorem 3.

Since Theorem 1 is false, as we have shown above, Theorem 3 cannot be true either because it clearly says more than what Theorem 1 does.

The above comments clearly debunk Theorems 1 and 3 of Dewan and Hans. 
2.1. The principal error in the proofs of Theorems 1 and 3. Since Theorems 1 and 3 are invalid, there must be something wrong with their proofs. This had to be looked into, which we did. We found a serious mistake in the proof of Lemma 1 of their paper [2]. It is applied to obtain Lemma 2, which the authors use to prove Theorem 1 . Here is what Lemma 1 of Dewan and Hans says.

Lemma 1. If $P(z)=c_{0}+\sum_{\nu=\mu}^{n} c_{\nu} z^{\nu}, 1 \leq \mu<n$ is a polynomial of degree $n$, having all its zeros in the disk $|z| \leq k, k \geq 1$, then for $|z|=1$

$$
k^{n+\mu-3}\left|Q^{\prime}(z)\right| \leq\left|P^{\prime}\left(k^{2} z\right)\right|,
$$

where $Q(z)=z^{n} \overline{P(1 / \bar{z})}$.

The polynomial $P(z):=z^{n}+k^{n}$ satisfies the conditions of Lemma 1 with any $\mu$ such that $1 \leq \mu<n$. For this polynomial, (6) reduces to $k^{\mu-1} \leq 1$, which clearly does not hold for any $\mu>1$ if $k>1$. This shows that Lemma 1 is false for $2 \leq \mu \leq n-1$ and $k>1$.

The authors use the faulty Lemma 1 to prove Lemma 2, stated as follows.

Lemma 2. If $P(z)=c_{0}+\sum_{\nu=\mu}^{n} c_{\nu} z^{\nu}, 1 \leq \mu<n$ is a polynomial of degree $n$, having all its zeros in the disk $|z| \leq k, k \geq 1$, then

$$
\max _{|z|=1}\left|Q^{\prime}(z)\right| \leq k^{n-\mu+1} \max _{|z|=1}\left|P^{\prime}(z)\right|,
$$

where $Q(z)=z^{n} \overline{P(1 / \bar{z})}$.

The example $z^{n}+k^{n}$ shows that this lemma is also false for $2 \leq \mu \leq n-1$ and $k>1$.

We note that the proof of Theorem 1, as given by Dewan and Hans, uses Lemma 2. Since Lemma 2 is deduced from Lemma 1, it is desirable to identify the error in the proof of Lemma 1 as presented by the authors on pages $57-58$ of [2]. So, we shall do that.

Using a standard argument, the authors conclude that ([2, p. 58], see

$$
k^{n-1}\left|Q^{\prime}(z / k)\right| \leq k\left|P^{\prime}(k z)\right| \text { for }|z| \geq 1 .
$$

This is fine. Since $c_{1}=\cdots=c_{\mu-1}=0$, this can be written as

$$
k^{n-1}\left|Q^{\prime}(z / k)\right| \leq k\left|(k z)^{\mu-1} \sum_{\nu=\mu}^{n} \nu c_{\nu}(k z)^{\nu-\mu}\right| \text { for }|z| \geq 1 .
$$

In particular, the authors say (see inequality (2.4) of their paper) that

$$
k^{n-1}\left|Q^{\prime}(z / k)\right| \leq k^{\mu}\left|\sum_{\nu=\mu}^{n} \nu c_{\nu}(k z)^{\nu-\mu}\right|
$$

for $|z|=1$. We agree with this. Next, they say that $\sum_{\nu=\mu}^{n} \nu c_{\nu}(k z)^{\nu-\mu} \neq 0$ in $|z|>1$ and we agree once again. Then they make the bizarre assertion 
that by maximum modulus principle it (by which they mean (7)) also holds for $|z|>1$. They overlook that for this to be true

$$
\frac{k^{n-1}\left|Q^{\prime}(z / k)\right|}{k^{\mu}\left|\sum_{\nu=\mu}^{n} \nu c_{\nu}(k z)^{\nu-\mu}\right|}
$$

must tend to a finite limit as $z \rightarrow \infty$. Except in the case where $c_{0}=0$ the above mentioned quotient tends to infinity as $z \rightarrow \infty$. Thus, the proof of Lemma 1 is based on a false application of the maximum modulus principle.

We are sorry to add that the authors apply Lemma 2 to prove another lemma which they state as follows.

Lemma 3. If $P(z)=c_{n} z^{n}+\sum_{\nu=\mu}^{n} c_{n-\nu} z^{n-\nu}, 1 \leq \mu<n$ is a polynomial of degree $n$, having no zeros in the disk $|z| \leq k, k \leq 1$, then

$$
k^{n-\mu+1} \max _{|z|=1}\left|P^{\prime}(z)\right| \leq \max _{|z|=1}\left|Q^{\prime}(z)\right|,
$$

where $Q(z)=z^{n} \overline{P(1 / \bar{z})}$.

Once again, the example $z^{n}+k^{n}$ shows that this lemma is also invalid for $2 \leq \mu \leq n-1$ and $k<1$.

3. Another related paper. The authors have gone on to use their faulty Lemmas 1, 2 and 3 in another paper, namely [1] published in Ann. Univ. Mariae Curie-Skłodowska Sect. A in the year 2011. As we shall explain, Theorems 1 and 2 of [1] are not true. The results in [1] involve the notion of polar derivative. The polar derivative of a polynomial $P(z)$ with respect to a point $\alpha$, denoted by $D_{\alpha} P(z)$, is defined by

$$
D_{\alpha} P(z)=n P(z)+(\alpha-z) P^{\prime}(z) .
$$

Theorem 1 of [1] can be stated as follows. Because of its obvious relationship with Theorem 2 of [2], stated above as Theorem 2, we shall name it Theorem $2 \mathrm{~b}$.

Theorem 2b. If $P(z)=c_{n} z^{n}+\sum_{j=\mu}^{n} c_{n-j} z^{n-j}, 1 \leq \mu<n$, is a polynomial of degree $n$ having all its zeros on $|z|=k, k \leq 1$, then for every real or complex number $\alpha$ with $|\alpha| \geq k$, we have

$$
\begin{aligned}
& \max _{|z|=1}\left|D_{\alpha} P(z)\right| \\
& \leq \frac{n\left(|\alpha|+k^{\mu}\right)}{k^{n-\mu+1}} \frac{n\left|c_{n}\right| k^{2 \mu}+\mu\left|c_{n-\mu}\right| k^{\mu-1}}{\mu\left|c_{n-\mu}\right|\left(1+k^{\mu-1}\right)+n\left|c_{n}\right| k^{\mu-1}\left(1+k^{\mu+1}\right)} \max _{|z|=1}|P(z)| .
\end{aligned}
$$

For $P(z):=z^{n}+k^{n}, k \leq 1$, which is a polynomial satisfying the conditions of Theorem $2 \mathrm{~b}$, inequality (8) says that

$$
k^{n}+|\alpha| \leq \frac{k^{\mu}+|\alpha|}{k^{n-\mu+1}} \frac{k^{2 \mu}}{k^{\mu-1}\left(1+k^{\mu+1}\right)}
$$


and so a fortiori $k^{n-2 \mu}\left(k^{n}+|\alpha|\right) \leq k^{\mu}+|\alpha|$, that is

$$
k^{2 n-2 \mu}+k^{n-2 \mu}|\alpha| \leq k^{\mu}+|\alpha| \text {. }
$$

If $k<1$, then $k^{2 n-2 \mu}>k^{\mu}$ and $k^{n-2 \mu}>1$ for any $\mu>2 n / 3$. Thus, (9) and so also (8) cannot hold for any $\mu>2 n / 3$. As indicated by the authors (see [1, pp. 6-7, §3]) the proof of Theorem $2 \mathrm{~b}$ uses Lemma 2 of [1], which is the same as Lemma 3 of [2], cited above. Since Lemma 3 of [2] is false, as we have already indicated, their proof of Theorem $2 \mathrm{~b}$ is invalid and there is really no need to look for counter-examples to (8) for $\mu \leq 2 n / 3$.

4. The polynomials considered by Dewan and Hans. It seems that Dewan and Hans overlooked the fact that in inequality (1) of Govil, equality holds for $P(z):=z^{n}+k^{n}$, which is a polynomial of the form $P(z):=$ $c_{0}+\sum_{\nu=\mu}^{n} c_{\nu} z^{\nu}$. To think that they could improve upon (1), by considering polynomials which are of the form $P(z):=c_{0}+\sum_{\nu=\mu}^{n} c_{\nu} z^{\nu}$, was not a promising idea to start with. They could obtain a stronger conclusion than that of Theorem A only if they considered a class of polynomials which did not contain the polynomial $z^{n}+k^{n}$. In fact, there is no raison d'ètre for Theorems 1 and 3 . Not only are their proofs not correct, their statements are false. The problem with Theorem 2 is of a different nature; its proof uses Lemma 3, which is faulty.

\title{
REFERENCES
}

[1] Ahuja, A., Dewan, K. K., Hans, S., Inequalities concerning polar derivative of polynomials, Ann. Univ. Mariae Curie-Skłodowska Sect. A 65 (2011), 1-9.

[2] Dewan, K. K., Hans, S., On maximum modulus for the derivative of a polynomial, Ann. Univ. Mariae Curie-Skłodowska Sect. A 63 (2009), 55-62.

[3] Govil, N. K., On the derivative of a polynomial, Proc. Amer. Math. Soc. 41 (1973), $543-546$.

[4] Govil, N. K., On a theorem of S. Bernstein, J. Math. Phys. Sci. 14 (1980), 183-187.

[5] Rahman, Q. I., Schmeisser, G., Analytic Theory of Polynomials, Clarendon Press, Oxford, 2002.

\author{
M. A. Qazi \\ Department of Mathematics \\ Tuskegee University \\ Tuskegee, Alabama 36088 \\ U.S.A. \\ e-mail: qazima@aol.com
}

Received May 12, 2013 